\title{
Fern-synthesized nanoparticles in the fight against malaria: LC/MS analysis of Pteridium aquilinum leaf extract and biosynthesis of silver nanoparticles with high mosquitocidal and antiplasmodial activity
}

\begin{abstract}
Malaria remains a major public health problem due to the emergence and spread of Plasmodium falciparum strains resistant to chloroquine. There is an urgent need to investigate new and effective sources of antimalarial drugs. This research proposed a novel method of fern-mediated synthesis of silver nanoparticles (AgNP) using a cheap plant extract of Pteridium aquilinum, acting as a reducing and capping agent. AgNP were characterized by UVï vis spectrophotometry, Fourier transform infrared (FTIR) spectroscopy, energydispersive X-ray spectroscopy (EDX), and X-ray diffraction (XRD). Phytochemical analysis of P. aquilinum leaf extract revealed the presence of phenols, alkaloids, tannins, flavonoids, proteins, carbohydrates, saponins, glycosides, steroids, and triterpenoids. LC/MS analysis identified at least 19 compounds, namely pterosin, hydroquinone, hydroxy-acetophenone, hydroxy-cinnamic acid, 5, 7-dihydroxy-4-methyl coumarin, trans-cinnamic acid, apiole, quercetin 3-glucoside, hydroxy-L-proline, hypaphorine, khellol glucoside, umbelliferose, violaxanthin, ergotamine tartrate, palmatine chloride, deacylgymnemic acid, methyl laurate, and palmitoyl acetate. In DPPH scavenging assays, the IC50 value of the P. aquilinum leaf extract was $10.04 \varepsilon \mathrm{g} / \mathrm{ml}$, while IC50 of BHT and rutin were 7.93 and $6.35 \mathrm{\varepsilon g} / \mathrm{ml}$. In mosquitocidal assays, LC50 of P. aquilinum leaf extract against Anopheles stephensi larvae and pupae were 220.44 ppm (larva I), $254.12 \mathrm{ppm}$ (II), $302.32 \mathrm{ppm}$ (III), $395.12 \mathrm{ppm}$ (IV), and $502.20 \mathrm{ppm}$ (pupa). LC50 of P. aquilinum-synthesized AgNP were $7.48 \mathrm{ppm}$ (I), 10.68 ppm (II), 13.77 ppm (III), 18.45 ppm (IV), and $31.51 \mathrm{ppm}$ (pupa). In the field, the application of P. aquilinum extract and AgNP (10何C50) led to $100 \%$ larval reduction after $72 \mathrm{~h}$. Both the $\mathrm{P}$. aquilinum extract and AgNP reduced longevity and fecundity of An. stephensi adults. Smoke toxicity experiments conducted against An. stephensi adults showed that P. aquilinum leaf-, stem-, and root-based coils evoked mortality rates comparable to the permethrin-based positive control $(57,50,41$, and $49 \%$, respectively). Furthermore, the antiplasmodial activity of P. aquilinum leaf extract and green-synthesized AgNP was evaluated against CQ-resistant (CQ-r) and CQ-sensitive (CQ-s) strains of P. falciparum. IC50 of P. aquilinum were 62.04 $\varepsilon \mathrm{g} / \mathrm{ml}$ (CQ-s) and $71.16 \mathrm{\varepsilon g} / \mathrm{ml}$ (CQ-r); P. aquilinum-synthesized AgNP achieved IC50 of $78.12 \mathrm{gg} / \mathrm{ml}$ (CQ-s) and $88.34 \mathrm{\varepsilon g} / \mathrm{ml}$ (CQ-r). Overall, our results highlighted that fernsynthesized AgNP could be candidated as a new tool against chloroquine-resistant $\mathrm{P}$. falciparum and different developmental instars of its primary vector An. stephensi. Further research on nanosynthesis routed by the LC/MS-identified constituents is ongoing.
\end{abstract}

Keyword: Anophelinae; Antioxidant; Fecundity; Longevity; Mosquito-borne diseases; Nanosynthesis; Nanotechnology; Smoke toxicity 